murine thymus assayed by quantitative hybridization of arrayed cDNA clones. Genomics 29:207-216.

7.Piétu, G., O. Alibert, V. Guichard, B. Lamy, F. Bois, E. Leroy, R. Mariage-Samson, R. Houlgatte et al. 1996. Novel gene transcripts preferentially expressed in human muscles revealed by quantitative hybridization of a high density cDNA array. Genome Res. 6:492-503.

8.Schena, M., D. Shalon, R.W. Davis and P.O. Brown. 1995. Quantitative monitoring of gene expression patterns with a complementary DNA microarray. Science 270:467-470.

9.Velculescu, V.E., L. Zhang, B. Vogelstein and K.W. Kinzler. 1995. Serial analysis of gene expression. Science 270:484-487.

10.Welle, S., K. Bhatt and C.A. Thornton. 1999. Inventory of high-abundance mRNAs in skeletal muscle of normal men. Genome Res. 9:506-513.

11.Zhao, N., N. Hashida, N. Takaashi, Y. Misumi and Y. Sakaki. 1995. High density cDNA filter analysis: a novel approach for large scale quantitative analysis of gene expression. Gene 156:207-213.

This work was supported by the Centre National de la Recherche Scientific (CNRS) and grants from Association Française contre les Myopathies (AFM). C.D. acknowledges the Fédération Française des Groupements Parkinsoniens (FFGP) for fellowships. We thank Marie-Dominique Devignes for useful discussions. Address correspondence to Dr. Geneviève Piétu, CNRS-ERS 1984, 19 rue Guy Moquet, BP 8, 94801 Villejuif Cedex, France. Internet: pietu@infobiogen.fr

Received 31 December 1998; accepted 12 July 1999.

Charles Decraene, Isabelle Reguigne-Arnould, Charles Auffray and Geneviève Piétu CNRS

Villejuif, France

\section{RT-PCR: Characteriza- tion of Long Multi-Gene Operons and Multiple Transcript Gene Clusters in Bacteria}

BioTechniques 27:966-972 (November 1999)

\section{ABSTRACT}

Reverse transcription (RT)-PCR is a valuable tool widely used for analysis of gene expression. In bacteria, RT-PCR is helpful beyond standard protocols of northern blot RNA/DNA hybridization (to identify transcripts) and primer extension (to locate their start points), as these methods have been difficult with transcripts that are low in abundance or unstable, similar to long multi-gene operons. In this report, RT-PCR is adapted to analyze transcripts that form long multi-gene operons-where they start and where they stop. The transcripts can also be semiquantitated to follow the expression of genes under different growth conditions. Examples using RT-PCR are presented with two different multi-gene systems for metal cation resistance to silver and mercury ions. The silver resistance system [9 open reading frames (ORFs); $12.5 \mathrm{~kb}$ is shown by RT-PCR to synthesize three nonoverlapping messenger RNAs that are transcribed divergently. In the mercury resistance system (8 ORFs; $6.3 \mathrm{~kb}$ ), all the genes are transcribed in the same orientation, and two promoter sites produce overlapping transcripts. For RT-PCR, reverse transcriptase enzyme is used to synthesize first-strand CDNA that is used as a template for PCR amplification of single-gene products, from the beginning, middle or end of long multi-gene, multi-transcript gene clusters.

\section{INTRODUCTION}

Reverse transcriptase catalyzes the synthesis of single-stranded DNA from RNA templates and is used in the laboratory primarily for the construction of cDNA libraries. Reverse transcriptionpolymerase chain reaction (RT-PCR) involves the synthesis of first-strand cDNA from mRNA templates, followed by PCR amplification using the first-strand cDNA as template. RT-PCR can detect rare transcripts (copy number as low as 1-100 per bacterial cell) and is used for detection and measurement of gene expression and for the diagnosis of infectious agents or genetic diseases $(4,5)$.

The experimental strategy described below provides an efficient method for the identification of long multi-gene bacterial operons and quantitation of expression of different genes. In bacteria, this has been a difficult process when using the conventional northern hybridization technique because of transcript stability and abundance. This report presents the technique with examples from transcripts of two different multi-gene systems that have been analyzed in detail by this new approach $(2,3)$. The silver resistance operon has 3 mRNAs transcribed divergently, while the mercury resistance system mRNAs are transcribed in the same orientation. Reverse transcriptase enzyme is used to synthesize full-length cDNA products from long mRNA species. This approach overcomes the need for optimization of PCRs to enable the synthesis of long PCR products corresponding to the first strand cDNA. Single-gene PCR products are the eventual products for analysis.

\section{MATERIALS AND METHODS}

Escherichia coli J53 (pMG101), Bacillus cereus RC607 and E. coli JM109 (pYW33) cells were grown in Luria-Bertani (LB) liquid broth under inducing or noninducing conditions for the set of genes that confer resistance to $\mathrm{AgNO}_{3}$ or $\mathrm{HgCl}_{2}$. Total RNA was isolated from these cells using the RNeasy ${ }^{\circledR}$ Extraction Kit (Qiagen, Valencia, CA, USA) and treated with RNase free DNase (Life Technologies, Gaithersburg, MD, USA) to eliminate contaminating DNA. For RT, $1 \mu \mathrm{g}$ of total cellular RNA was used for the synthesis of first-strand cDNA using SUPERSCRIPT $^{\text {TM }}$ II Reverse-Transcriptase enzyme (Life Technologies). The RT reactions $(20 \mu \mathrm{L})$, per the manufacturer's recommendations, were conducted at $42^{\circ} \mathrm{C}$ for $60 \mathrm{~min}$. Then, 1.5 $\mu \mathrm{L}$ of the RT reaction product mixture was used as template for PCR after 
treatment with RNase H. PCR amplification was carried out in the presence of $1.5 \mathrm{mM} \mathrm{MgCl} 2$ with 30 cycles at $94^{\circ} \mathrm{C}$ for $30 \mathrm{~s}$, at $55^{\circ} \mathrm{C}$ for $30 \mathrm{~s}$, at $72^{\circ} \mathrm{C}$ for $5 \mathrm{~min}$ (for $E$. coli silver resistance), at $94^{\circ} \mathrm{C}$ for $30 \mathrm{~s}$, at $50^{\circ} \mathrm{C}$ for $30 \mathrm{~s}$ and at $72^{\circ} \mathrm{C}$ for $3 \mathrm{~min}$ (for mercury resistance system in B. cereus and $E$. coli). The PCR was carried out using Taq DNA Polymerase (Life Technologies) and amplification products were analyzed by agarose gel electrophoresis and stained with ethidium bromide.

\section{RESULTS AND DISCUSSION}

The first system to be analyzed by this RT-PCR technique was the silver resistance system of plasmid pMG101 in $E$. coli $\left(\right.$ GenBank ${ }^{\circledR}$ Accession No. AF067954) (2). This included a set of nine genes and open reading frames (ORFs) designated silE, silS, silR, silC, ORF96, silB, silA, ORF 105 and silP in order. For ease of description and understanding in this report, these are called ORF1-ORF9, respectively, in Figure 1A. ORFs 1, 2 and 3 are transcribed divergent to ORFs 4-9 (2). The nine ORFs traverse $12.5 \mathrm{~kb}$ of DNA, and there were several possibilities (based on the direction of synthesis, spacing, sequence analysis for canonical patterns and known gene functions) for the synthesis of a maximum of five and perhaps a minimum of two transcripts (Figure 1A).

To determine which hypothesized transcripts were synthesized, RT-mediated, first-strand cDNA was synthesized using either primer 1, 2, 3 or 4 (Figure 1A) and total cellular RNA from $\mathrm{Ag}^{+}$-induced cells as template. Primers 1, 2, 3 and 4 correspond to the 3 ' region of ORFs 1, 2, 4 and 9, respectively. The cDNA synthesized served as template for the PCR amplification of individual single ORFs. The combination of RT and PCR oligonucleotide primers used are shown in Figure 1A. We used a cDNA template synthesized with RT-primer 1 for PCR amplification with primer pairs PCR1/PCR2 and PCR3/PCR4; cDNA synthesized with RT-primer 2 for PCR amplification with primer pairs PCR3/PCR4 and PCR5/PCR6; cDNA synthesized with RT-primer 3 for PCR amplification with primer pair PCR3/PCR4 and cDNA synthesized with RT-primer 4 for PCR amplification with primer pairs PCR5/PCR6 and PCR7/PCR8.

In PCRs, using gene-specific primers, in which cDNA from $\mathrm{RT}$ reaction with RT-primer 1 was used, the DNA amplification product was obtained for ORF1 (Figure 2A, lane 3) but not ORF3 (2, data not shown). This result established that ORF1 and ORF3 are synthesized on separate transcripts, although their orientation allowed for the hypothesis of a single longer co-transcript. In PCRs with cDNA from the RT reaction with RT-primer 2, an amplification product was obtained for ORF3 (Figure 2A, lane 2) but not ORF4 (data not shown). This showed that ORFs 2 and 3 were synthesized as a single transcript, and that ORF3 and ORF4 were synthesized on separate transcripts. ORF3 was also not amplified by PCR when cDNA from the RT reaction with RT-primer 3 was used (data not shown). In PCRs with the product of the RT reaction using RTprimer 4 as template, ORF4 was amplified (Figure 2A, lane 1). In a separate PCR with the product of RT reaction using RT-primer 4, the amplification product for ORF9 was equivalent in amounts to the product for ORF4 described earlier (2, data not shown). These results demonstrated that ORFs 4-9 are synthesized as a single long transcript of $9.5 \mathrm{~kb}(2)$. The three transcripts were also mapped by primer extension and northern hybridization (2), and no additional smaller transcripts as hypothesized in Figure 1A were seen. Since the conditions for RT-PCR were constant in these experiments, the differing amounts of PCR products (Figure 2A) suggest the relative cellular abundance of the three mRNAs (silE/ORF1 more than silSR/ORFs 2 and 3, which in turn was more abundant than the transcript for silCORF96BAORF105P/ORFs 4 through 9; see Figure 2A and Reference 2).

The second system used to develop RT-PCR for long bacterial transcripts was that for mercury resistance of the gram-positive bacterium $B$. cereus RC607 (GenBank Accession No. AF138877) (3). With this system, quantitation and inducibility of transcript synthesis were also measured. The mer 


\section{Short Technical Reports}

operon of B. cereus RC607 includes a set of eight chromosomal genes and ORFs designated merR1, merT, ORF3, ORF4, merA, merR2, merB2 and merB1 in order (Figure 1B) (3). Similar to the silver resistance system described here, these genes and ORFs are called ORF1-ORF8, respectively. The chromosomal mercury resistance system of $B$. cereus was cloned and expressed also on a plasmid in $E$. coli, which allows comparisons of the same mRNA products produced in both gram-positive and -negative bacteria. Such a system might synthesize one or two transcripts (Figure 1B). The purpose of the experiments described was to identify the number of transcripts and to compare the expression of individual genes with RNA isolated from cells grown under induced (growth for $60 \mathrm{~min}$ in a medium supplemented with $\mathrm{HgCl}_{2}$ ) and un-induced conditions. Total RNA was isolated from $\mathrm{Hg}^{2+}$-induced and un-in- duced cultures of $B$. cereus and $E$. coli and used as template for RT reactions, using RT-primer 1 or RT-primer 2 (Figure $1 \mathrm{~B})$, corresponding to regions near the 3 ' ends of merA/ORF5 and merB1/ ORF8, respectively.

Additional RT oligonucleotide primers that corresponded to the $3^{\prime}$ ends of the standardization control genes (for 16S rRNA) from $B$. cereus and $E$. coli were included in the same RT reactions as mercury gene-specific RT-primers 1

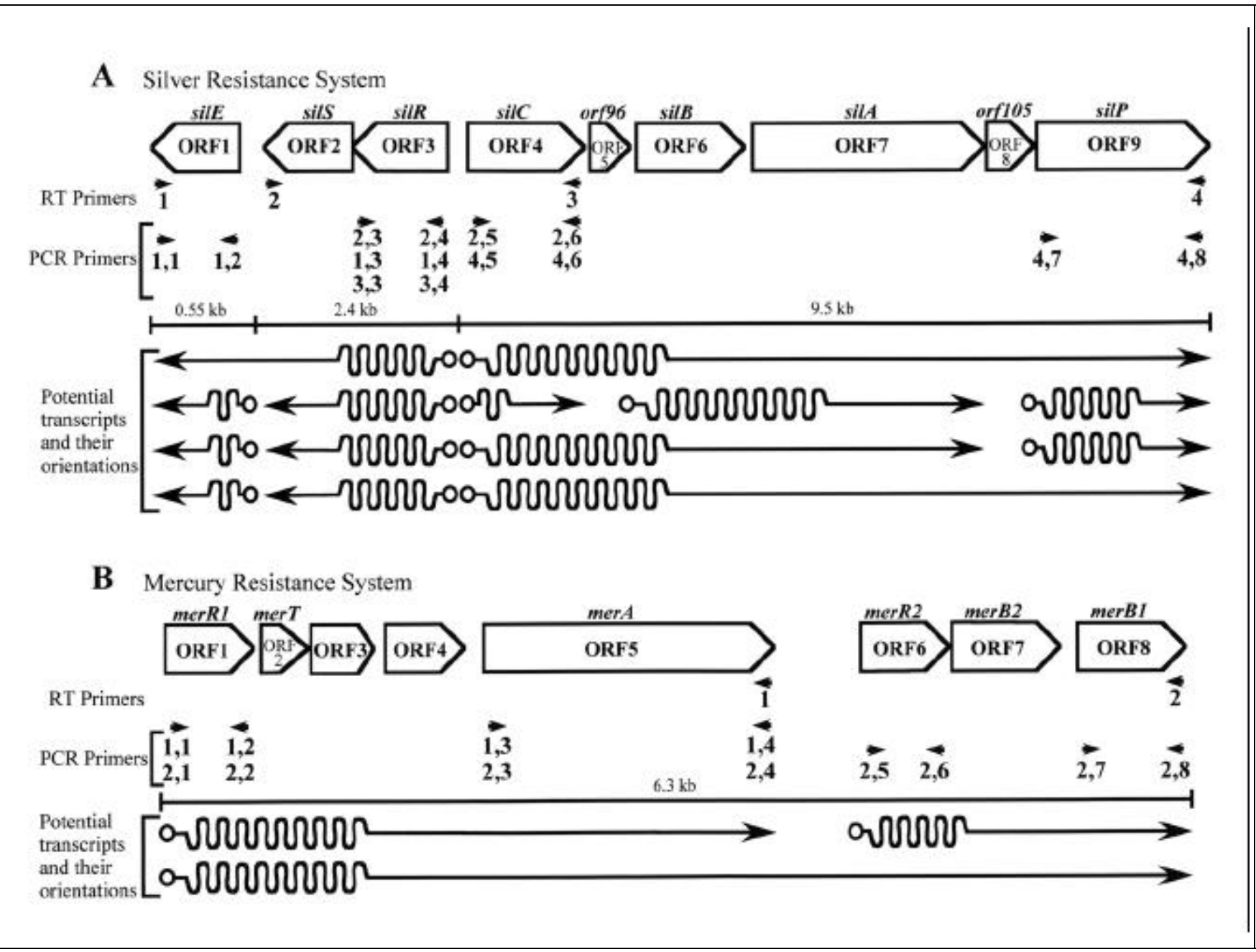

Figure 1. Diagrams of two multi-gene metal resistance systems. (A) Silver resistance in E. coli and (B) mercury resistance in B. cereus RC607. Lengths are given in kilobase pairs $(\mathrm{kb})$; potential transcripts and positions of oligonucleotide primers used for RT reactions and PCR are shown. Arrowheads show the positions, $5^{\prime}$ to $3^{\prime}$ directions of oligonucleotide primers or proposed mRNA products. Oligonucleotide primers used for RT reactions and PCR are denoted by numbers, and the combination of RT reactions and PCR primers used is shown. For PCR primers, the first number refers to the RT primer used for the RT reaction (that provides the cDNA template for PCR), and the second number refers to the PCR primers. For example, 2,3 means that RT reaction with RT-primer 2 is used to provide the cDNA template and PCR primer 3 is one of the primers used for amplification. The sequence of oligonucleotide primers in $5^{\prime}$ to $3^{\prime}$ orientation are: (A) for the silver resistance system, RT1 and PCR1: GGCCAGACTGACCGTTATTCATAA; RT2: GTTTGCTGCATGACAGGCTAATGACATC; RT3 and PCR6: TAAATTTACTCTACCCAACCGC; RT4 and PCR8: CGGGCAGACCAGCAATAACAGATA; PCR2: GTACTCCCCCGGACATCACTAATT; PCR3: AGCGAGTGAGAAAGGTAGTCT; PCR4: AACAGGTGAATATCTCAGCAAG; PCR5: CTCAGCATCAGTACCATATTCATC; PCR7: CATGACATATCCTGAAGACAGAAAATGC and (B) for the mercury resistance system, RT1 and PCR4: ATCCAGCACAGCAAGATAAT; RT2 and PCR8: ATTGGCAGGTCTAGATTCCT; PCR1: GTTAACAAGGAGACCATTCG; PCR2: TCAGTGTTTCAATAATGGGG; PCR3: CGATTGAAGTAGATTTTCGC; PCR5: GAGGATTTAACGATTGGTCA; PCR6: GGAATCAAGGTACGATTCAA; PCR7: AATCGTAACCCGACTTGAC. 


\section{Short Technical Reports}

or 2. The products of the RT reactions were templates for amplification of individual ORFs by PCR. The combination of RT and PCR oligonucleotide primers used are given in Figure 1B. We used a cDNA template synthesized with RT-primer 1 for PCR amplification with primer pairs PCR1/PCR2 and PCR3/PCR4; and cDNA synthesized with RT-primer 2 for PCR amplification with primer pairs PCR1/PCR2, PCR3/ PCR4, PCR5/PCR6 and PCR7/PCR8.

With RNA isolated from $B$. cereus cells and cDNA synthesized from RT primer 1, ORFs 1 and 5 were amplified by PCR (Figure 2B). This shows that ORFs 1 through 5 were synthesized as a single transcript. The products of ORF1 and ORF5 were less abundant in the uninduced than the induced. ORFs 1 and 5 were also amplified from $E$. coli total cellular RNA with cDNA from RT with RT-primer 1. However, the amounts of PCR products amplified for the un-in- duced and induced cell RNAs were essentially the same in E. coli (Figure 2B). PCR products were not found in control reactions where RNA was treated with RNase or when the $\mathrm{RT}$ reaction was run without RT enzyme.

Using cDNA from RT with RTprimer 2 (Figure 1B), PCR products were amplified for ORFs 5, 6 and 8 (Figure 2B), which established that mercury resistance ORFs 5, 6, 7 and 8 are synthesized as a single transcript. With RT-primer 2, ORF1 is also amplifiable by PCR (3), showing that all eight ORFs are co-transcribed as a single transcript of $6.3 \mathrm{~kb}$. With RNA from Bacillus using RT product with RTprimer 2, the amount of the PCR products for ORF5 and ORF8 were more with the induced cells than with the uninduced cells (Figure 2B). There was no significant indication of inducibility with PCR amplification of ORFs 5, 6 and 8 in the $E$. coli background (Figure
2B). Equivalent amounts of RT-PCR products for the $16 \mathrm{~S}$ rRNA genes from $B$. cereus and $E$. coli were found in the induced and un-induced (Figure 2C), showing that equivalent amounts of RNA were taken for the reactions.

The results reported here and in References 2 and 3 show that this RT-PCR strategy can be effectively used for the identification, characterization and, at least semiquantitation of long multigene operons in bacteria. First, RT-PCR establishes which ORFs are included and excluded from a given transcript. In addition, the approach can be used for the analysis of inducible expression of different ORFs, as tested with the mercury resistance system. However, the results of such measurements need to be carefully interpreted in view of all the usual caveats: gene function, inducible or constitutive expression, posttranscriptional processing and stability, initiation of transcription or the pres- 


\section{Short Technical Reports}

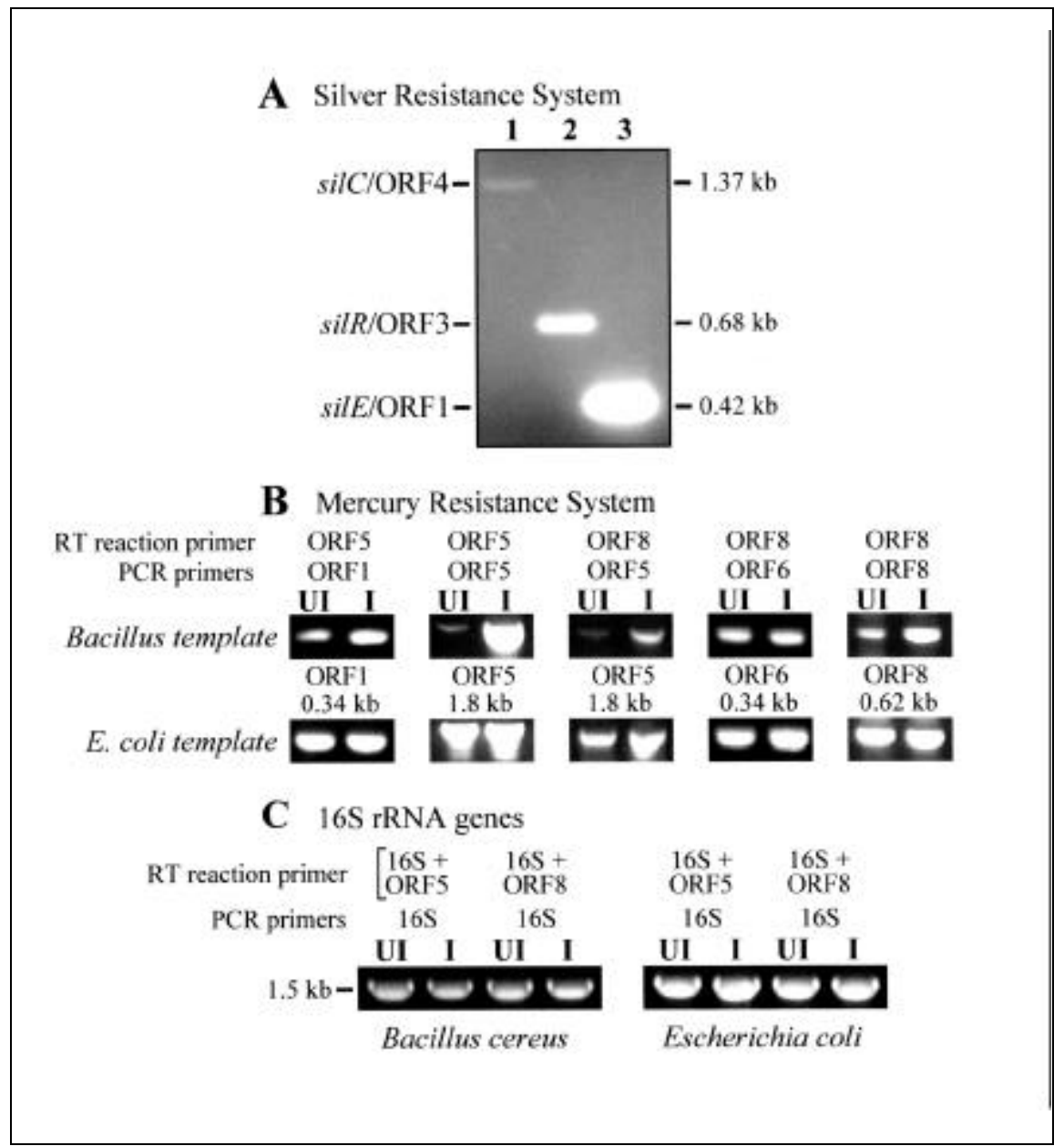

Figure 2. PCR amplification products for different ORFs of (A) silver resistance determinant, (B) mercury resistance determinant and $(C) 16 S$ ribosomal RNA genes, analyzed on agarose gels and stained with ethidium bromide. (A) The PCR amplification products of silC/ORF4 (lane 1), silR/ORF3 (lane 2) and silE/ORF1 (lane 3) using cDNA products generated with RT-primers 4, 2 and 1, respectively. (B) The PCR amplification products of ORFs 1, 5, 6 and 8, using cDNA products generated with RT-primers 1 and 2, with total cellular RNA extracted from un-induced or induced (grown in the presence of $\left.\mathrm{HgCl}_{2}\right)$ B. cereus and E. coli cells. The ORFs amplified and the sizes of PCR products [in kilobase $(\mathrm{kb})$ pairs] are given. (C) The PCR products for the $16 \mathrm{~S}$ rRNA genes (used as controls) amplified from the same RT reactions as with mercury oligonucleotide RT-primers 1 and 2 and total RNA from B. cereus and E. coli. Panel A has been modified from Reference 2 with permission. Gene expression can be quantitated by measuring the intensity of PCR products (in pixels from digitized camera images), comparing the RNA from un-induced and induced cells.

ence of "imperfect" transcription stop sites (3). For example, Gambill and Summers (1) showed a transcriptional gradient in a different mercury resistance operon and that rates of mRNA synthesis varied from one end of the operon to the other. In that mer operon, mRNA degradation rates also varied, but only slightly for different genes.

The RT-PCR strategy developed here is powerful because the PCR products are well defined (Figure 2) and readily quantified. Furthermore, the experiments require relatively little start- ing material and do not involve elaborate reaction optimizations.

\section{REFERENCES}

1.Gambill, B.D. and A.O. Summers. 1992. Synthesis and degradation of the mRNA of the Tn21 mer operon. J. Mol. Biol. 225:251259

2.Gupta, A., K. Matsui, J.-F. Lo and S. Silver. 1999. Molecular basis for resistance to silver cations in Salmonella. Nat. Med. 5:183-188.

3.Gupta, A., L.T. Phung, L. Chakravarty and S. Silver. 1999. Mercury resistance in Bacillus cereus RC607: transcriptional organization and two new open reading frames. J. Bacteriol. 181(22), in press

4.Pregliasco, F., C. Mensi, L. Camorali and G. Anselmi. 1998. Comparison of RT-PCR with other diagnostic assays for rapid detection of influenza viruses. J. Med. Virol. 56:168-73.

5.Sellner, L.N. and G.R. Turbett. 1998. Comparison of three RT-PCR methods. BioTechniques 25:230-234.

I thank Simon Silver for support and critical feedback. This research was supported by a DOE grant and research funds from the University of Illinois. Address correspondence to Dr. Amit Gupta, Department of Microbiology and Immunology, University of Illinois at Chicago, M/C 790, Room 703, 835 South Wolcott Avenue, Chicago, IL 60612-7344, USA. Internet: agupta@uic.edu

Received 24 March 1999; accepted 6 July 1999.

\section{Amit Gupta \\ University of Illinois \\ College of Medicine \\ Chicago IL, USA}

